\title{
Proof of principle: The adaptive geometry of social foragers
}

\author{
Marcus J. Dostie ${ }^{a, b}$, David Lusseau ${ }^{c}$, Tyler Bonnell ${ }^{b, d}$, Parry M. R. Clarke ${ }^{b}$, George Chaplin ${ }^{\mathrm{e}}$, Stefan \\ Kienzle $^{\mathrm{a}, \mathrm{b}}$, Louise Barrett ${ }^{\mathrm{b}, \mathrm{d}}$ and S. Peter Henzi* b,d
}

\author{
a Department of Geography, University of Lethbridge, 4401 University Drive, Lethbridge T1K 3M4, Canada. \\ ${ }^{\mathrm{b}}$ Applied Behavioural Ecology and Ecosystems Research Unit, The University of South Africa Private Bag X6, Florida 1710, \\ South Africa. \\ c Institute of Biological and Environmental Sciences, University of Aberdeen, Aberdeen AB24 2TZ, UK. \\ ${ }^{\mathrm{d}}$ Department of Psychology, University of Lethbridge, 4401 University Drive, Lethbridge T1K 3M4, Canada. \\ ${ }^{\text {e }}$ Department of Anthropology, Pennsylvania State University, University Park, PA 16802, USA.
}

* Corresponding author: Department of Psychology, University of Lethbridge, 4401 University Drive, Lethbridge T1K 3M4, Canada. Telephone: +1-403 327-6835. Fax: +1-403 329-2555. peter.henzi@,uleth.ca

The spatial configuration of a group of animals should reflect the ability of its members to respond to environmental contingencies. Under predation risk, the optimal position for an individual in a stationary group is at the group's centre. The resulting group geometry is circular, with individual placement determined by competitive ability. Where it compromises efficient foraging, a long-standing question has been whether this topology can deform adaptively in response to the local distribution of resources. Here we show that the shape described by a group of foraging baboons changes in response to habitat structure and that this promotes foraging efficiency while conserving the predation-risk-related distribution of group members. Adult baboons improve unimpeded access to the small, dispersed food items found in grassland by adjusting both their inter-individual distances and their relative positions along the line of movement in order to forage in rank formation. Dominant animals occupy the centre of the group and do so regardless of its geometry. Our results demonstrate that spatially explicit data can address emergent group-level properties directly. This global approach complements analyses of individual action and can help direct the search for potential local rules of interaction.

Keywords: Collective action, Papio hamadryas, predation risk, selfish herd, spatial structure 
Principle Ten. A rank foraging formation will be favored whenever there is an advantage to remaining in a group and the group is foraging on slowly renewing resources that are of low overall density in the home range and are not locally abundant.

S.A. Altmann 1974 (p. 241).

The spatial configuration of a group of animals is the summation of the responses of its members to the local environment, made under the constraint of association (Parrish \& Edelstein-Keshet 1999). In mobile groups this emergent geometry is expected to deform adaptively as group members accommodate to local shifts in the relative salience of competing costs (Beecham \& Farnsworth 1999; Morrell, Ruxton, \& James 2011).

Social species, such as primates, that form groups to reduce the risk of predation for their members (Hill \& Dunbar 1998; Shultz, Opie, \& Atkinson 2012) do so at the expense of increasing local competition for resources (van Schaik 1983; Majolo, de Bortoli Vizioli, \& Schino 2008). Heterogeneity in the distribution of these risks and costs both among group members (Ron, Henzi, \& Motro 1996; Koenig 2002) and across the landscape (Willems \& Hill 2009), makes such groups well suited to investigating the environmental drivers of the spatial structure of social units (Krause \& Ruxton, 2002; Morrell et al. 2011).

Under marginal predation, where animals on the edge of groups are more vulnerable to predators, those that are closer to the group's centre have smaller domains of danger and are less exposed to risk (Hamilton 1971; Morrell \& Romey 2008; King et al. 2012). Local adjustments in response to risk perception will then generate a group geometry that ideally, in two dimensions, is circular (Aurenhammer 1991), with individual location determined by resource holding potential (Parker 1974), which can be indexed as dominance rank. This configuration is likely to characterise the global structure of animal groups primarily where high quality resources are clumped and can be defended, and marginal animals can balance increased predation risk against the possibility of improved foraging opportunities offered by a reduction in contest competition (Krause \& Ruxton, 2002; Bumann, Krause, \& Rubenstein 1997; Robinson 1981). The question, then, is whether the accommodation of this 'selfish herd' (Hamilton 1971) to changing cost potentials (Beecham \& Farnsworth 1999) can be detected at the global level as a change in adaptive topology?

Savanna baboons (Papio hamadryas) have home ranges that encompass habitat mosaics and frequently forage in grasslands (Henzi \& Barrett 2005), which are typified by low quality, thinly dispersed, quickly consumed foods (Henzi, Byrne, \& Whiten 1992). These induce scramble competition (Isbell 1991), where the persistence of a circular formation would reduce foraging efficiency for all but those animals in the group's vanguard (Hirsch 2007). In 1974, Altmann (1974) instantiated and extended Hamilton's general theoretical argument to predict that in grassland, where ephemeral resources are thinly distributed, group members will spread out to secure at least the minimum foraging swath that enables unimpeded foraging while adjusting their positions to keep abreast of their neighbours and thereby minimize the costs of scramble competition. The geometry of this foraging group is expected, therefore, to deform to a rank formation as animals encounter low quality, dispersed resources, while the spread of the group will be constrained by continuing predation risk. As an implicit but necessary corollary, we also expect more dominant animals to continue to avoid the margins of the group as the shape shifts.

An assessment of these long-standing propositions can contribute to the development of a coherent group-level spatial ecology that complements current research on individual contributions to collective movement (Parrish \& Edelstein-Keshet 1999; Nagy et al. 2010; Katz et al. 2011; Sumpter, Mann, \& Perna 2012; Strandburg-Peshkin et al. 2015), but has had to wait on the emergence of appropriate global positioning system (GPS) technologies and geospatial analytics (Tomkiewicz et al. 2010). Here, we use interpolated GPS data collected from individual adults to describe dynamic spatial relationships in a group of chacma baboons as it moves through its home range, which comprises a mix of open grassland and dense scrub (Kotze \& Fairall 2006).

\section{METHODS}

\section{Study site and data collection}

We collected 74 days of data - spread across 391 days from a habituated group of chacma baboons $(\mathrm{N} \approx 45)$ at De Hoop Nature Reserve, South Africa (Barrett et al. 2004) during 2007 and early 2008. A single observer, using a handheld GPS-equipped data logger followed and recorded the spatial locations of all 14 adult members $\left(\mathrm{N}_{\text {Males }}=3\right.$, $\mathrm{N}_{\mathrm{Females}}=11$ ). On-site calibration of the data loggers confirmed that they were absolutely accurate to within 2-5 $\mathrm{m}$ and relatively accurate to $\sim 1 \mathrm{~m}$ (viz. the accuracy with which the distance between two points can be estimated). Beginning at one end of the group, the observer identified, stood next to, and collected a GPS record for each visible adult in turn. When the distal end of the group was reached, the observer turned back along the line of travel while continuing to collect data. If an animal was not seen during two circuits of the troop, the observer interrupted data collection in order to locate it. We obtained 61,842 usable data points, with a mean of 63.98 points $(+/-9.03 \mathrm{SD}) /$ individual/day. Foraging effort was determined from scan sample records of activity $(\mathrm{N}=5846)$, collected as standard procedure (Henzi et al. 2009) every $30 \mathrm{~min}$ from all visible, identified individuals, and expressed as the proportion of the group foraging. Animals were assigned an ordinal dominance rank derived from ad libitum records of all dyadic agonistic interactions over the study period, with participants identified as winners or losers. We used the Domicalc program (Schmidt \& De Vries) in R 3.2.1. (R Core Team, 2015) to confirm a high degree of linearity in the hierarchy $\left(\mathrm{h}^{1}=1 ; N=704 ; P<0.0001\right)$. 
Ethical note: All procedures were approved by the University of Lethbridge Animal Welfare Committee (Protocol \#0702).

\section{Interpolation and estimates of minimal optimal spread}

While our spatial data collection procedure did not allow the simultaneous recording of all individual locations, the median time interval of $7 \mathrm{~min}$ between consecutive records for the same individual preserves sufficient information for analyses derived from individual points (Andrienko et al. 2013; Bonnell, Henzi, \& Barrett 2015), giving us confidence in the use of interpolated data. We therefore estimated the positions of all group members for any given time using linear interpolation of trajectories between successive point samples. To confirm that our baboons were sensitive to both predation risk and foraging interference, we followed the approach detailed in Aureli et al (2012), extracting the inter-individual distances (IIDs. N=5204. Range: $0 \mathrm{~m}-615 \mathrm{~m})$ of all animals at $11 \mathrm{am}$ each day, when animals were consistently likely to be foraging, and fitting them to three models, with model selection based on the Akaike Information Criterion (AIC). These models were derived from the relative ability of (i) food distribution and predation risk (the Ecological model), (ii) foraging interference (the Biosocial model) or (iii) both (the Socioecological model) to predict observed IIDs. In summary, the Ecological model is an integrated estimate of the spatial distribution of important ecological factors (predation risk, food) in the habitat, with individual distribution following a Poisson process in response to this background heterogeneity. The Biosocial model assumes that individual distribution is governed solely by repulsion from others at close distances and attraction towards them at intermediate distances. The Socioecological model, therefore, is an extension of the Biosocial model that accounts explicitly for the ecological landscape (see Aureli et al. 2012 for detail and derivations) in predicting individual distributions. It should be noted that, for these analyses, we make the simplifying assumption that the extracted distances of repulsion and attraction do not vary significantly across habitats and individual group members.

The Socioecological model provided the best fit to the data and consequently we doubled its estimate of the mean distance at which animals repelled one another to set the width of the swaths within which each animal might forage without interference. The degree to which individual swaths overlapped, estimated as a proportion of the distance along the $\mathrm{x}$-axis (Figure 1), constituted our group-level estimate of the extent to which group spread reflected the predicted minimal requirements for unimpeded individual foraging in open country. The lower the overlap in foraging swaths, the more optimally the group was foraging. Where there was overlap in foraging swaths, we distinguished between animals in front (no immediate scramble competition) from those further back, who might be expected to encounter less food as a consequence of foraging in another animal's path.

\section{Data location error and temporal independence}

We constrained the data set to periods for which the average location error was predicted to be at or below $7.8 \mathrm{~m}$, using a model of interpolation error derived from separately collected continuous tracks of individual baboons (Dostie et al. in prep.). This model estimated interpolation error by repeated subsampling of the continuous data and comparing the interpolated data generated in this way with the continuous data. We also measured autocorrelation in the dependent variable (i.e. group-level minimal optimal spread) by fitting a semi-variogram with the gstat package (Pebesma 2004) in R. Point estimates of optimal foraging spread separated by $15 \mathrm{~min}$ offered the best trade-off between independence and sample size. Using these criteria, we were able to extract 349 group point clouds representing the estimated location of every individual at a particular moment.

\section{Speed, Direction of Travel, Rotation, and Centring of Groups}

Direction of travel (DoT) and speed of these group estimates were measured at the group level using the line between the group centroids (i.e. the mean $x, y$ location of the group) at Time $t$ and $t+1$. To simplify the measurement of group optimal spread across multiple samples with variable DoT orientations, group point clouds were rotated so that their DoT was set to $0^{\circ}$. In order to rotate the group point cloud, it was first centred on the Cartesian plane with the group centre at $(0,0)$. This was done by subtracting the group mean centre from each individual's point. The point cloud was then rotated by applying the following formula to individual points within the centred point cloud:

$X_{r}=X_{z} \cos R-Y_{z} \sin R: Y_{r}=X_{z} \sin R+Y_{z} \cos R$

Where:

$X_{r}$ and $Y_{r}=$ Rotated $\mathrm{x}$ and $\mathrm{y}$ values

$X_{z}$ and $Y_{z}=$ The pre-rotation $\mathrm{x}$ and $\mathrm{y}$ values

$R=$ Rotation in radians (difference between direction of travel and 0)

\section{Group geometry}

A rank formation refers to one in which individuals are spread out along an $\mathrm{x}$-axis which is perpendicular to the direction of travel. We estimated the extent to which group shape approximated a rank formation using the "Directional Distribution (Standard Deviational Ellipse)" tool in ArcGIS10.2, (ESRI, 2011) to generate ellipses around the group point clouds at each independent sample (Figure 1). Ellipses specified at different standard distances (SD) are centred at the same point, oriented in the same direction and retain the same proportions. Consequently, we arbitrarily bounded ellipses at $2 \mathrm{SD}$ to extract measurements with which to determine group shape. We used the orientation of the ellipse (i.e., the direction of the long axis) and the short and long axes to estimate rank formation as follows:

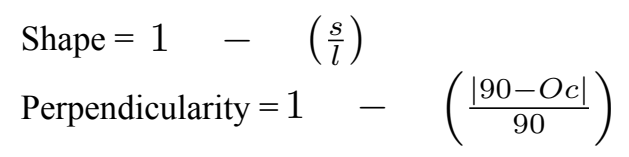

Group formation $=$ Shape $*$ Perpendicularity 
Where:

$\mathrm{s}=$ length of short axis

$1=$ length of long axis

$\mathrm{O}_{\mathrm{e}}=$ Orientation of the long axis in relation to the direction of travel $\left(0^{\circ}\right.$ to $\left.180^{\circ}\right)$

While the resulting values that approach 0 can indicate either circles or files, only formations that are both linear and perpendicular to the DoT can approach 1.

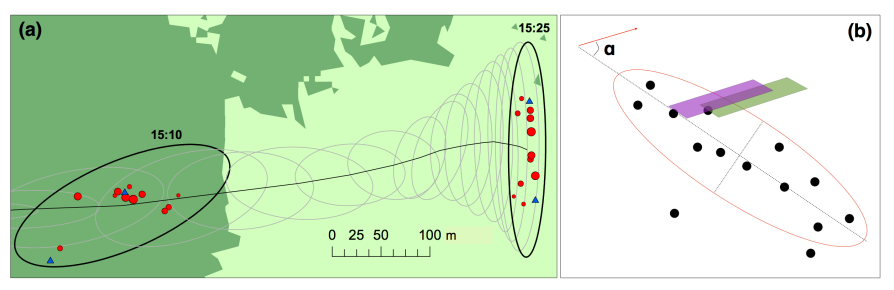

Fig. 1. Transformation of baboon group geometry. (a) Shift to rank formation as the group moves from dense (dark green) scrub to grassland (light green). Individual group point clouds (Females: red circles; Males: Blue triangles) were used to generate ellipses, bounded for illustrative purposes at $2 \mathrm{SD}$, at 1 min intervals (grey), while analysis was restricted to those that were $15 \mathrm{~min}$ apart (black), with time indicated. The black line indicates travel direction. Larger circles reflect increasing female dominance rank. (b) Schematic of elements used to estimate group shape and foraging spread (see Methods). Dashed lines: short and long axes of ellipse; Purple and green bars: minimal expected optimal foraging swaths, illustrating suboptimal overlap for the purple swath; $\alpha$ : angle of orientation relative to travel direction (indicated by the red line).

\section{Location within the group}

Each animal's distance to the centre of the group (DtC) was expressed as its Euclidean distance to the centre of the ellipse.

\section{Habitat classification}

At the time of the study, the home range consisted of dense fynbos scrub, recently burnt areas and grassland (Kotze \& Fairall 2006). This made it appropriate to classify the habitat as either closed (0) or open (1) and we used interactive, supervised classification in ArcGIS 10.2 (ESRI, 2011) to allocate each pixel in a high resolution digital aerial photograph of the home range to one of the two categories. Training areas were assigned using geo-referenced photographs in conjunction with ground-truthing at the site. By overlaying point clouds on the photograph we could assign, for each independent sample in the analysis, a habitat category to each subject's position. These were then used to generate a proportional measure, ranging between 0 and 1, for the group as a whole.

\section{Statistical analyses}

Models were run in Stata 14 (StataCorp, 2015) and, after corroboration of the outcomes, goodness-of-fit was estimated for the LMM (Johnson 2014) with the MuMin package (Barton
$\&$ Barton 2015) in R. Tests were two-tailed with $\alpha$ level set to 0.05 . All continuous independent variables were centred. Test assumptions were controlled for by computation of variance inflation factors (all VIF <1.2) and visual inspection of scatterplots, histograms, and Q-Q plots of residuals.

\section{RESULTS}

\section{Distances of attraction and repulsion}

After appropriate spatial interpolation, we modeled IIDs among troop members to assess the dimensionality of interindividual interactions (Aureli et al. 2012). The Socioecological model $(\triangle \mathrm{AIC}=0)$, which models forces of attraction (a) and repulsion (r) among group members while accounting for environmental heterogeneity, explained IID distribution better than either the Biosocial $(\triangle \mathrm{AIC}=403)$ or the simple Ecological model $(\triangle \mathrm{AIC}=2743.2$. Table 1$)$. As required by the predictions (Altmann 1974), therefore, these baboons were spatially constrained by both a reluctance to be too far away (model mean estimate: $\mathrm{a}=71.3 \mathrm{~m}$ ) or too close (model mean estimate: $\mathrm{r}=3.9 \mathrm{~m}$ ) to others.

Table 1. Parameter estimates for the three competing models of interindividual spacing.

\begin{tabular}{lccc}
\hline Parameter & Socioecological model $(95 \mathrm{Cl})$ & Biosocial model & Ecological model \\
\hline $\mathrm{pa}$ & $0.0002(0.0002-0.0002)$ & $0.0002(0.0002-0.0002)$ & \\
$\mathrm{a}$ & $71.3(70.8-71.8)$ & $73(72.5-73.5)$ & \\
$\mathrm{pr}$ & $0.065(0.003-0.127)$ & & \\
$\mathrm{r}$ & $3.9(1.9-5.9)$ & $0.44(0.43-0.45)$ & \\
$\beta$ & $3.8(1.75-5.86)$ & & $2.6(2.2-3.0)$ \\
$\mathrm{AIC}$ & -3740 & -3591 & -0.997 \\
\hline
\end{tabular}

pa: proportional contribution of attraction; $\mathbf{p}_{\mathbf{r}}$ : proportional contribution of repulsion a: range of attraction; $\mathbf{r}$ : range of repulsion (both in metres); $\boldsymbol{\beta}$ is the inverse of $\lambda$, the mean of the spatial Poisson process explaining the distribution of individuals. The estimated $95 \%$ confidence intervals for each parameter are provided in parentheses; AIC: Akaike Information Criterion.

\section{Foraging spread, group shape and habitat}

We used the minimal foraging swath $(2 \mathrm{r}=7.8 \mathrm{~m})$ and the extent of overlap of individual swaths as a group-level estimate of minimal optimal foraging spread, where less overlap indicates improved foraging for the group as a whole. (Fig. 1). As Altmann's principle expects group shape to promote efficiency in baboons foraging on widelydispersed, low-quality food items, we entered minimum optimal foraging spread as the response variable and habitat type, group formation and the proportion of the group foraging (indexing foraging effort) as predictors in a fullfactorial linear model, with travel speed specified as a control variable. The results indicate that minimum optimal foraging spread was independently and positively associated with more open habitat, increasing rank formation, the proportion of the group that was foraging, as well as with the three-way interaction of these variables (Table 2). The 
foraging spread of our baboon group was therefore most likely to meet minimum requirements for optimality when it was in rank formation and foraging in open terrain (Figure 2).

Table 2. Minimal optimal foraging spread as a function of habitat structure, foraging effort and group formation.

\begin{tabular}{|c|c|c|c|c|c|}
\hline Parameter & $\beta$ & SE & t Ratio & $P$ & $95 \% \mathrm{Cl}$ \\
\hline Speed & 0.003 & 0.001 & 5.060 & $<.0001$ & $0.002-0.004$ \\
\hline Habitat structure & 0.130 & 0.019 & 6.720 & $<.0001$ & $0.092-0.168$ \\
\hline Foraging effort & 0.089 & 0.031 & 2.900 & 0.004 & $0.029-0.150$ \\
\hline Group formation & 0.405 & 0.038 & 10.770 & $<.0001$ & $0.331-0.479$ \\
\hline Habitat ${ }^{\star}$ Foraging effort & 0.008 & 0.090 & 0.090 & 0.928 & $-0.169-0.185$ \\
\hline Habitat ${ }^{\star}$ Formation & $\begin{array}{c}-0.09 \\
9\end{array}$ & 0.114 & -0.870 & 0.385 & $-0.323-0.125$ \\
\hline Foraging effort ${ }^{\star}$ Formation & 0.247 & 0.164 & 1.500 & 0.134 & $-0.076-0.570$ \\
\hline Habitat*Formation*Foraging effort & 1.146 & 0.532 & 2.150 & 0.032 & $0.099-2.193$ \\
\hline Intercept & 0.166 & 0.022 & 7.640 & $<.0001$ & $0.123-0.209$ \\
\hline
\end{tabular}

Whole model: $N=349$; F-ratio $=30.897 ; \mathrm{DF}_{8,340} ; P<0.001$. Adj. $R^{2}=0.407$.
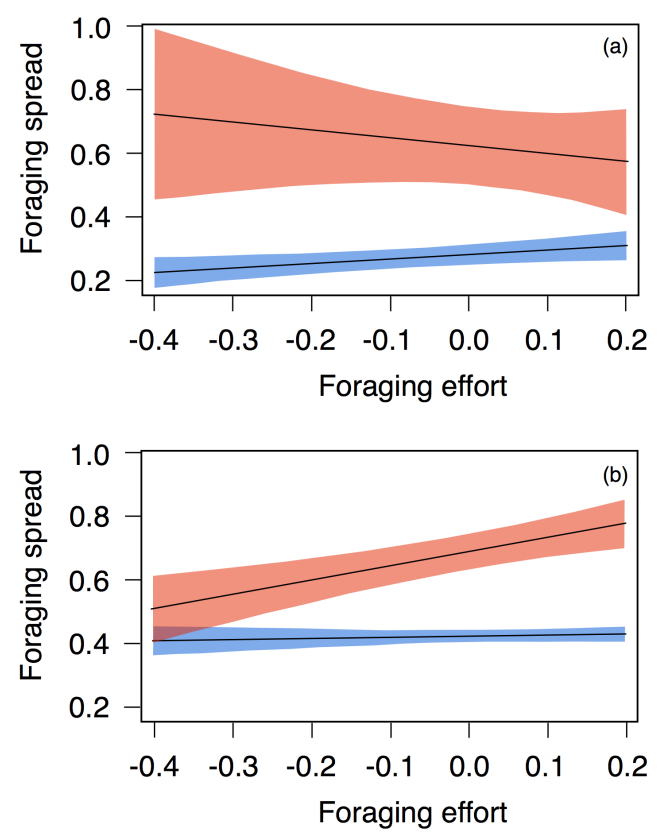

Fig. 2. Modeling foraging spread. The relationship between the extent of minimum optimality in foraging spread and foraging effort (the number of animals foraging) in (a) Closed habitat and (b) Open habitat. Model predictions $(+/-95 \mathrm{CI})$ in each are presented for high (red) and low (blue) rank formation.

\section{Dominance rank and position within the group}

We used a linear mixed model (LMM) to test the hypothesis that high-ranking animals were more likely to be closer to the group centre regardless of group geometry. We applied a square root transform to the dependent variable to improve the fit to normality. All adult chacma baboon males are dominant to all females (Henzi \& Barrett 2003), subordinate males avoid the alpha male (Bonnell et al. in prep.) while, at twice the size of females, males are also less likely to avoid the margins of the group (Rhine, Bioland, \& Lodwick 1985). We therefore specified subject sex as a control variable and dominance rank and group formation as fixed effects (with interaction term). We specified subject ID as a random effect, with a random slope for the effect of spatial formation. With sex controlled, the results indicated that higher-ranking animals were closer to the centre of the group regardless of group geometry (Table 3 . Figure 3 ).

Table 3. Distance from the group centre as a function of dominance and group shape.

\begin{tabular}{lccccc}
\hline Parameter & $\beta$ & SE & $\mathrm{Z}$ & $P$ & $95 \% \mathrm{Cl}$ \\
\hline Sex (Ref: Female) & 1.172 & 0.185 & 6.340 & 0.000 & $0.809-1.534$ \\
Dominance rank & 0.097 & 0.019 & 5.180 & 0.000 & $0.060-0.133$ \\
Group shape & -0.740 & 0.394 & -1.880 & 0.060 & $-1.511-0.031$ \\
Rank ${ }^{\star}$ Shape & 0.081 & 0.045 & 1.820 & 0.069 & $-0.006-0.169$ \\
Intercept & 3.489 & 0.175 & 19.930 & 0.000 & $3.146-3.832$
\end{tabular}

Whole model: $N=4243$. Log likelihood $=-8429.493$, Wald $X^{2}{ }_{4}=$ 52.40; $P<0.0001 . R^{2}$ Marginal $=0.045 ; R^{2}$ Conditional $=0.058$.

\section{DISCUSSION}

Our results indicate that the shape described by a group of baboons as it traverses its home range conforms to that expected of a mobile selfish herd engaging adaptively with changing cost potentials in the environment (Beecham \& Farnsworth 1999; Altmann 1974). Shifting priorities can therefore account for the absence of the file formation expected of a moving group under predation risk alone (Bumann et al. 1997). Topologically, the configuration of adults in the study troop was homeomorphic, with dominance rank determining individual location within each sex regardless of group shape. At the same time, highranking females were closer to the group's centre than were males. In general, therefore, this result confirms Altmann's expectations while also pointing to a need to consider more particularly the relative importance of other factors, such as vulnerability and reproductive competition, in explaining individual decisions about spatial location. It is probable, for instance, that the effect of rank on centrality in males is driven by male-male competition and therefore only coincidentally mirrors the effect in females, which is more likely to be a consequence of perceived predation risk.

That said, it is clear that the goodness-of-fit of the model accounting for individual positioning is markedly poorer than that of the model describing foraging efficiency in relation to group shape. While this will be due partly to interpolation error and the coarse-grained characterisation of the environment (Josephs et al. in press), we believe that 
the disparity reflects accurately the conditions faced by baboons at this field site. That is, while predators able to target baboons occur, they do so in very low numbers, and we have no evidence of either attempted or successful predation (Barrett et al. 2004). Not surprisingly, perceived risk, as indexed by the relatively large distance at attraction $(71.3 \mathrm{~m})$, is also low. Given this, the effect size in the configuration model serves as a gauge of perceived predation risk and we would anticipate it being larger in populations where predation is a more prominent source of mortality. At De Hoop, however, it seems that the cost potential encountered by the baboons as they move derives primarily from the effect of resource dispersion, with predation risk, given the absence of an interaction between centrality and group geometry, exerting a consistent but low-level, background effect on individual location, if only primarily for females.

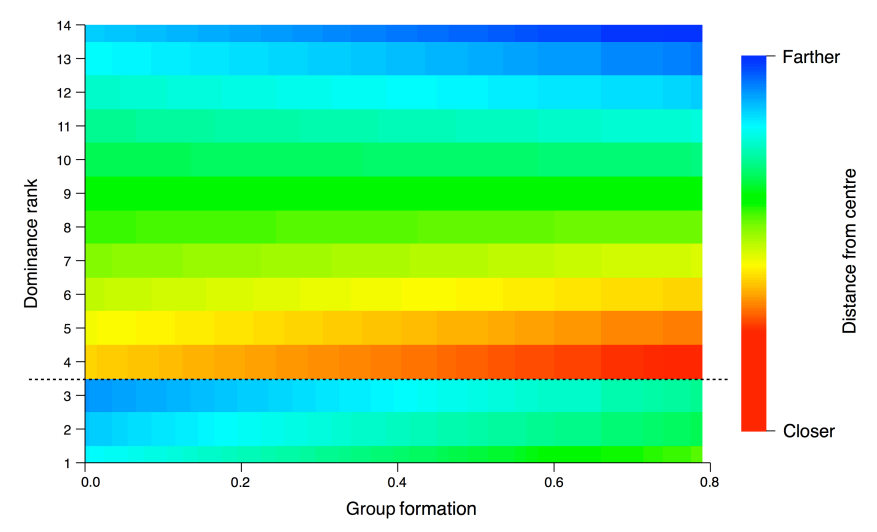

Fig. 3. Dominance rank determines spatial location. The heat map indicates the LMM fit to the data for distance to the group centre in relation to rank (highestranking animal $=1$ ) and group shape (rank formation $=1.0$ ). The dashed line separates males (ranks $1-3$ ) from females. Regardless of group shape, males were further from the group centre than high-ranking females and higherranking females were closer to the centre of the group than lower-ranking females.

This outcome confirms predictions of the emergence of adaptive shape (Parrish \& Edelstein-Keshet 1999) that were derived explicitly at the level of the group. Taken together, group geometry, together with the particular individual configurations that describe it, serves as a global indicator of individual decision-making without any a priori prescription as to the form of the decisions themselves. In so doing, it confirms the utility of a global-to-local trajectory in the modeling of collective behaviour (Sumpter et al. 2012). It also offers the more general prospect that such sparse data sets, which are logistically simpler to assemble than the highresolution equivalents generated by animal-mounted devices (Bonnell et al. 2015), can contribute to the development of this topic.

While not prescriptive, both the theory and the results generated at the global level certainly point to a circumscribed range of possible local rules that can be modeled and assessed empirically. The primary rules that describe spatial dynamics are those that govern the approach, avoidance and alignment of group members (Couzin et al. 2002; Eftimie et al. 2007), and a rule of preferential attraction to clusters of other group members has been shown to underpin collective movement and basal spatial coherence in baboons, both in Kenya (Strandburg-Peshkin et al. 2015), and at De Hoop (Bonnell et al. 2015). What our results suggest, however, is a need to consider the rules that are nested within this primary decision set that then govern the particular configurations of individuals under different ecological contexts. That is, while an undifferentiated attraction to the group's 'centre of mass' sustains spatial coherence and coordinated movement, subsequent positions are fine-tuned by rates at which food is encountered and the identities of neighbours, and constrained by the perceived risk of predation.

\section{ACKNOWLEDGEMENTS}

We thank Cape Nature for permission to undertake the study. We thank Dr Matt Grove and two anonymous referees for comments and suggestions that improved the manuscript substantially. This research was funded by grants from the Leakey Foundation, National Science and Engineering Research Council, Canada to S.P.H. and L.B., and by the National Research Foundation, South Africa to S.P.H. His co-authors dedicate this paper to the memory of P.M.R.C.

The authors declare no competing interests.

\section{REFERENCES}

Altmann, S. A. (1974). Baboons, space, time, and energy. American Zoologist, 14, 221-248.

Andrienko, N., Andrienko, G., Barrett, L., Dostie, M., \& Henzi, P. (2013). Space transformation for understanding group movement. IEEE Transactions on Visualization and Computer Graphics, 19, 2169-2178.

Aureli, F., Schaffner, C. M., Asensio, N., \& Lusseau, D. (2012). What is a subgroup? How socioecological factors influence interindividual distance. Behavioral Ecology, 23, 1308-1315.

Aurenhammer, F. (1991). Voronoi diagrams - a survey of a fundamental geometric data structure. ACM Computing Surveys (CSUR), 23, 345-405.

Barrett, L., Gaynor, D., Rendall, D., Mitchell, D., \& Henzi, S. P. (2004). Habitual cave use and thermoregulation in chacma baboons (Papio hamadryas ursinus). Journal of Human Evolution, 46, 215-222.

Barton, K., \& Barton, M. K. (2015). Package 'MuMIn'. Version, 1, 18. ftp://155.232.191.229/cran/web/packages/ MuMIn/

Beecham, J. A., \& Farnsworth, K. D. (1999). Animal group forces resulting from predator avoidance and competition minimization. Journal of Theoretical Biology, 198, 533-548. 
Bonnell, T. R., Henzi, S. P., \& Barrett, L. (2015). Sparse movement data can reveal social influences on individual travel decisions. arXiv preprint arXiv:1511.01536.

Bumann, D., Krause, J., \& Rubenstein, D. (1997). Mortality risk of spatial positions in animal groups: the danger of being in the front. Behaviour, 134, 1063-1076.

Couzin, I. D., Krause, J., James, R., Ruxton, G. D., \& Franks, N. R. (2002). Collective memory and spatial sorting in animal groups. Journal of Theoretical Biology, 218, 1-11.

Eftimie, R., de Vries, G., Lewis, M. A., \& Lutscher, F. (2007). Modeling group formation and activity patterns in selforganizing collectives of individuals. Bulletin of Mathematical Biology, 69, 1537-1565.

ESRI. (2011). ArcGIS Desktop: Release 10. Redlands, CA: Environmental Systems Research Institute.

Hamilton, W. D. (1971). Geometry for the selfish herd. Journal of Theoretical Biology, 31, 295-311.

Henzi, S. P., \& Barrett, L. (2005). The historical socioecology of savanna baboons (Papio hamadryas). Journal of Zoology, 265, 215-226.

Henzi, S. P., \& Barrett, L. (2003). Evolutionary ecology, sexual conflict, and behavioral differentiation among baboon populations. Evolutionary Anthropology, 12, 217-230.

Henzi, S. P., Byrne, R. W., \& Whiten, A. (1992). Patterns of movement by baboons in the Drakensberg mountains: primary responses to the environment. International Journal of Primatology, 13, 601-629.

Henzi, S. P., Lusseau, D., Weingrill, T., Schaik, C. P., \& Barrett, L. (2009). Cyclicity in the structure of female baboon social networks. Behavioral Ecology and Sociobiology, 63, 1015-1021.

Hill, R. A., \& Dunbar, R. I. M. (1998). An evaluation of the roles of predation rate and predation risk as selective pressures on primate grouping behaviour. Behaviour, 135, 411-430.

Hirsch, B. T. (2007). Costs and benefits of within-group spatial position: A feeding competition model. The Quarterly review of Biology, 82, 9-27.

Isbell, L. A. (1991). Contest and scramble competition: patterns of female aggression and ranging behavior among primates. Behavioral Ecology, 2, 143-155.

Johnson, P. C. D. (2014). Extension of Nakagawa \& Schielzeth's R2GLMM to random slopes models. Methods in Ecology and Evolution, 5, 944-946.

Josephs, N., Bonnell, T., Dostie, M.J., Barrett, L. \& Henzi, S.P. (In press). Working the crowd: Sociable vervets benefit by reducing exposure to risk. Behavioral Ecology.

Katz, Y., Tunstrøm, K., Ioannou, C. C., Huepe, C., \& Couzin, I. D. (2011). Inferring the structure and dynamics of interactions in schooling fish. Proceedings of the National Academy of Sciences, 108, 18720-18725.

King, A. J., Wilson, A. M., Wilshin, S. D., Lowe, J., Haddadi, H., Hailes, S., \& Morton, A. J. (2012). Selfish-herd behaviour of sheep under threat. Current Biology, 22, R561-R562.

Koenig, A. (2002). Competition for resources and its behavioral consequences among female primates. International Journal of Primatology, 23, 759-783.
Kotze, I. J. D. F., \& Fairall, N. (2006). Using Landsat TM imagery to map fynbos plant communities: a case study. South African Journal of Wildlife Research, 36, 75-87.

Krause, J., \& Ruxton, G. D. (2002). Living In Groups. Oxford: Oxford University Press.

Majolo, B., de Bortoli Vizioli, A., \& Schino, G. (2008). Costs and benefits of group living in primates: group size effects on behaviour and demography. Animal Behaviour, 76, 1235-1247.

Morrell, L. J., \& Romey, W. L. (2008). Optimal individual positions within animal groups. Behavioral Ecology, 19, 909-919.

Morrell, L. J., Ruxton, G. D., \& James, R. (2011). Spatial positioning in the selfish herd. Behavioral Ecology, 22, 16-22.

Nagy, M., Ákos, Z., Biro, D., \& Vicsek, T. (2010). Hierarchical group dynamics in pigeon flocks. Nature, 464, 890-893.

Parker, G. A. (1974). Assessment strategy and the evolution of fighting behaviour. Journal of Theoretical Biology, 47, 223-243.

Parrish, J. K., \& Edelstein-Keshet, L. (1999). Complexity, pattern, and evolutionary trade-offs in animal aggregation. Science, 284, 99-101.

Pebesma, E. J. (2004). Multivariable geostatistics in S: the gstat package. Computers \& Geosciences, 30, 683-691.

R Core Team. (2015). R: A Language and Environment for Statistical Computing. Vienna, Austria: R Foundation for Statistical Computing.

Rhine, R. J., Bioland, P., \& Lodwick, L. (1985). Progressions of adult male chacma baboons (Papio ursinus) in the Moremi Wildlife Reserve. International Journal of Primatology, 6, 115-122.

Robinson, J. G. (1981). Spatial structure in foraging groups of wedge-capped capuchin monkeys Cebus nigrivittatus. Animal Behaviour, 29, 1036-1056.

Ron, T., Henzi, S. P., \& Motro, U. (1996). Do female chacma baboons compete for a safe spatial position in a southern woodland habitat? Behaviour, 475-490.

Shultz, S., Opie, C., \& Atkinson, Q. D. (2012). Stepwise evolution of stable sociality in primates. Nature, 479, 219-222.

Schmid, V.S., \& de Vries, H. (2013). Finding a dominance order most consistent with a linear hierarchy: an improved algorithm for the ISI method. Animal Behaviour, 86, 1097-1105

StataCorp. (2015). Stata Statistical Software: Release 14. StataCorp, College Station, TX. StataCorp, College Station, TX.

Strandburg-Peshkin, A., Farine, D. R., Couzin, I. D., \& Crofoot, M. C. (2015). Shared decision-making drives collective movement in wild baboons. Science, 348, 1358-1361.

Sumpter, D. J. T., Mann, R. P., \& Perna, A. (2012). The modelling cycle for collective animal behaviour. Interface Focus, 2, 764-773.

Tomkiewicz, S. M., Fuller, M. R., Kie, J. G., \& Bates, K. K. (2010). Global positioning system and associated 
technologies in animal behaviour and ecological research. Philosophical Transactions of the Royal Society B: Biological Sciences, 365, 2163-2176.

van Schaik, C. P. (1983). Why are diurnal primates living in groups. Behaviour, 87, 120-144.

Willems, E. P., \& Hill, R. A. (2009). Predator-specific landscapes of fear and resource distribution: effects on spatial range use. Ecology, 90, 546-555. 